near the center (where there is a high probability of finding the electron and hole at the same relative position). Next $(t=250 \mathrm{fs})$, interference between the excitonic and free-carrier WP is seen. Because the WP is highly anisotropic, there is a net dipole moment that results in the $\mathrm{THz}$ spectrum shown in Figure 2. At later times ( $t=400,640 \mathrm{fs}$ ), side lobes can be seen in the WP; these are formed by the combination of slow transverse spreading, the relatively fast field driven motion in the polarization direction, and the excitonic attraction (Coulombic rescattering). The resulting WPs for the $\phi=\pi / 2$ case are significantly different. ${ }^{5}$

In conclusion, we have theoretically investigated the simultaneous exposure of an $\operatorname{In}_{x} \mathrm{Ga}_{1-x}$ As $\mathrm{QW}$ to a broadband optical pulse and a $1 \mathrm{THz}$ driving field. The scenario produces interfering, electron-hole WPs whose anisotropic beating structure can be manipulated to achieve harmonicemission in the $\mathrm{THz}$ regime. This technique may prove to be a useful means of generating tunable $\mathrm{THz}$ transients in the laboratory. Moreover, enhanced tunability has been very recently demonstrated via the selective excitation of spatially chosen WPs using two-pulse nondegenerate optical pulses. $^{7}$

\section{Acknowledgments}

This work was supported by NSF grant DMR9705403 and by the Office of Naval Research. The authors acknowledge R. Zimmerman for useful discussions.

\section{References}

1. S. Hughes and D.S. Citrin, "Ultrafast heating and switching of a semiconductor optical amplifier using half-cycle terahertz pulses," Phys. Rev. B. 58 (1998).

2. J.L. Krause et al., "Creating and detecting shaped Rydberg wavepackets," Phys. Rev. Lett. 79, 4978 (1998).

3. K.B. Nordstom et al., "Observation of dynamical Franz-Keldysh effect," Phys. Stat. Sol. b 204, 52 (1997). Using magnetoexcitons: J. Kono et al., "Resonant terahertz optical sideband generation from confined magnetoexcitons," Phys. Rev. Lett. 79, 1758 (1997).

4. A.P. Jauho and K. Johnsen, "Dynamical Franz-Keldysh effect," Phys. Rev. Lett. 24, 4576 (1996).

5. S. Hughes and D.S. Citrin, "The creation of highly anisotropic wavepackets in quantum wells: Dynamical Franz-Keldysh effect in the optical and terahertz regimes," Phys. Rev. B. 59 (P.R. 15969), (1999).

6. S.L. Chuang et al., "Optical rectification at semiconductor surfaces," Phys. Rev. Lett. 68, 102 (1992); P.C.M. Planken et al., "Terahertz emission in single quantum-wells after coherentoptical excitation of light hole and heavy hole excitons," Phys. Rev. Lett. 69, 3800 (1992).

7. S. Hughes and D.S. Citrin, "Two-pulse nondegenerate excitation of electron-hole wavepackets in quantum wells: Tunable terahertz emission," Opt. Lett., in press.

\title{
Quarter Wave Fresnel Rhomb with Colinear Output Beam
}

\section{A. Serpengüzel, Dept. of Physics, Bilkent Univ., Bilkent, Ankara, Turkey.}

\section{Abstract}

A standard quarter wave $(\lambda / 4)$ Fresnel rhomb laterally displaces the output beam with respect to the input beam. This lateral displacement can cause problems when inserting the rhomb in an already aligned optical setup. This note describes the use of two $\lambda / 8$ rhombs to construct a $\lambda / 4$ rhomb. The output of the proposed rhomb is colinear with the input beam, and allows a much more flexible optical alignment.

\section{Introduction}

Achromatic quarter wave $(\lambda / 4)$ or half wave $(\lambda / 2)$ retarders are manufactured using Fresnel rhombs. ${ }^{1}$ The standard $\lambda / 4$ rhomb creates a phase shift $(\delta)$ of $90^{\circ}$ between the parallel $(I /)$ and the perpendicular $(\perp)$ components of an incident polarized beam upon total internal reflection (TIR). If properly aligned, it can convert a linearly polarized beam to a circularly polarized beam and vice versa. The output of the $\lambda / 4$ rhomb is displaced laterally with respect to the input beam. This lateral displacement causes alignment problems, when inserting the rhomb in an already aligned optical setup.

The standard $\lambda / 2$ rhomb creates a phase shift $(\delta)$ of $180^{\circ}$ between the // and $\perp$ components of an incident polarized beam. If properly aligned, it can rotate a linearly polarized beam by $90^{\circ}$. The standard $\lambda / 2$ rhomb is composed of two $\lambda / 4$ rhombs. The output of the $\lambda / 2$ rhomb is colinear with the input beam, making the optical alignment much more flexible.

This note proposes a $\lambda / 8$ Fresnel rhomb that creates a phase shift $(\delta)$ of $45^{\circ}$ between the // and $\perp$ components of an incident polarized beam. The proposed $\lambda / 4$ Fresnel rhomb is composed of two $\lambda / 8$ rhombs. The output of the proposed $\lambda / 4$ rhomb is colinear with the input beam, making the optical alignment much more flexible.

\section{Calculation of the phase shift}

The working principle of the Fresnel rhomb is based on the phase shift between the // and $\perp$ components of an incident polarized beam upon TIR. This phase shift can be calculated using

$$
\delta=2 \tan ^{-1}\left\{\left\{\cos \left(\theta_{i}\right)\left[\sin ^{2}\left(\theta_{i}\right)-n^{-2}\right]^{1 / 2}\right\} / \sin ^{2}\left(\theta_{\mathrm{i}}\right)\right\},
$$

where $\theta_{i}$ is the angle of incidence and $n$ is the refractive index of the rhomb.

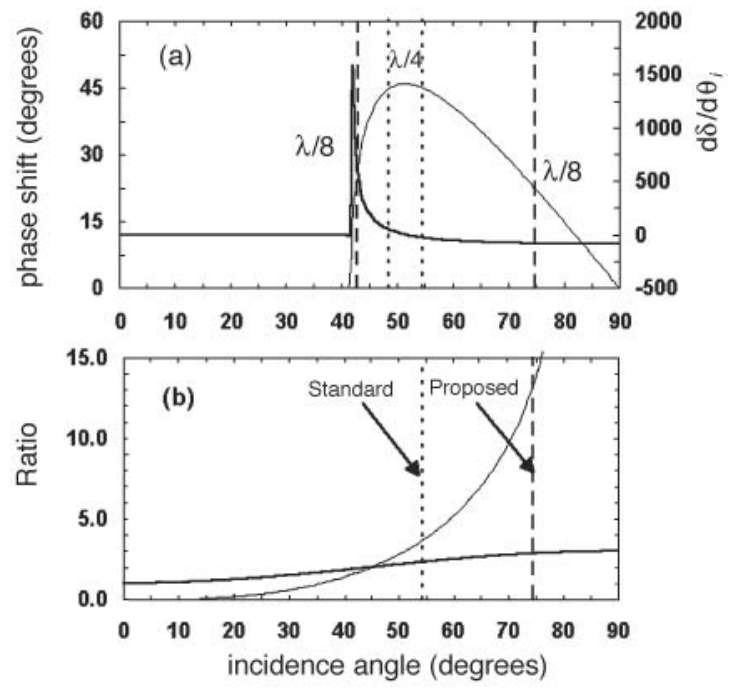

Figure 1. (a) Calculated phase shift (thin line) and its derivative (thick line) as a function of the incidence angle for a glass rhomb $(n=1.51$ ). (b) Calculated L/A (thin line) and H/A (thick line) ratios as a function of the incidence angle. 

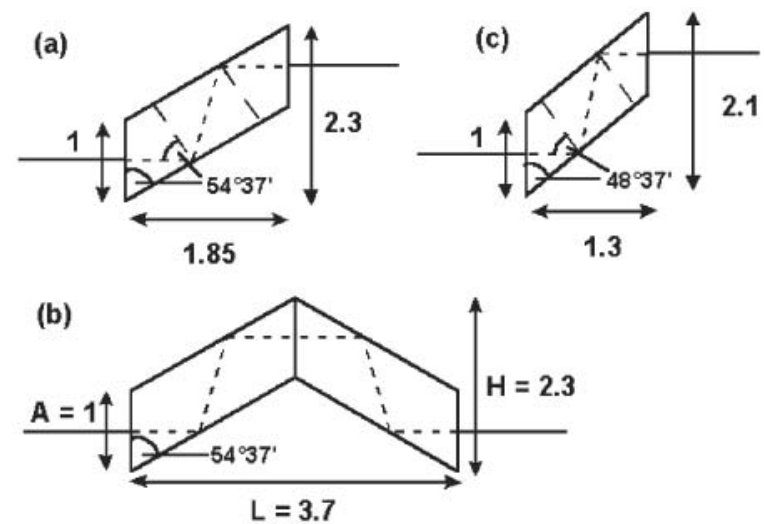

Figure 2. (a) Optical schematic of the (a) standard $\lambda / 4$, (b) standard $\lambda / 2$, and (c) unrealized $\lambda / 4$ rhombs.

Figure 1a (previous page) shows the phase shift, $\delta$, between the // and $\perp$ polarized waves as a function of the incidence angle for glass $(n=1.51)$ calculated using Eq. 1 . Figure la also shows the first derivative of $\delta$ with respect to the incidence angle $\left(\mathrm{d} \delta / \mathrm{d} \theta_{i}\right)$ to estimate the variation of $\delta$ for small angular misalignments, with larger variations corresponding to smaller angular tolerances.

\section{Calculation of the physical dimensions}

Figure $1 \mathrm{~b}$ shows the length/aperture $(\mathrm{L} / \mathrm{A})$ and the height/aperture $(\mathrm{H} / \mathrm{A})$ ratios of a double rhomb calculated using

$$
\begin{gathered}
\mathrm{L} / \mathrm{A}=4 \sin ^{2}\left(\theta_{\mathrm{i}}\right) \tan \left(\theta_{\mathrm{i}}\right), \\
\mathrm{H} / \mathrm{A}=1+2 \sin ^{2}\left(\theta_{\mathrm{i}}\right) .
\end{gathered}
$$

\section{The standard $\lambda / 4$ and $\lambda / 2$ rhombs}

As indicated by the dotted lines labeled $\lambda / 4$ in Figure 1a, a phase shift of $\lambda=45^{\circ}$ occurs for both $\left(\theta_{i}\right)=48^{\circ} 37^{\prime}$ (with $\mathrm{d} \delta / \mathrm{d} \theta_{i}=53$ ) and $\theta_{\mathrm{i}}=54^{\circ} 37^{\prime}$ (with $\mathrm{d} \delta / \mathrm{d} \theta_{i}=-27$ ). Since the angular tolerance of $\theta_{i}=54^{\circ} 37^{\prime}$ is higher than $\theta_{i}=48^{\circ} 37^{\prime}$, the standard $\lambda / 4$ rhomb uses two $\delta=45^{\circ}$ TIR at $\theta_{i}=54^{\circ} 37^{\prime}$. The standard $\lambda / 4$ rhomb is shown in Figure 2a. The rhomb angle is equal to the incidence angle of $\theta_{i}=54^{\circ} 37^{\prime}$. The output beam is displaced laterally, parallel to the input beam. As mentioned previously, this lateral displacement creates difficulties in optical alignment. For a standard $\lambda / 4 \mathrm{rhomb}$, a typical aperture size of $1 \mathrm{~cm}$ requires a length of $1.85 \mathrm{~cm}$
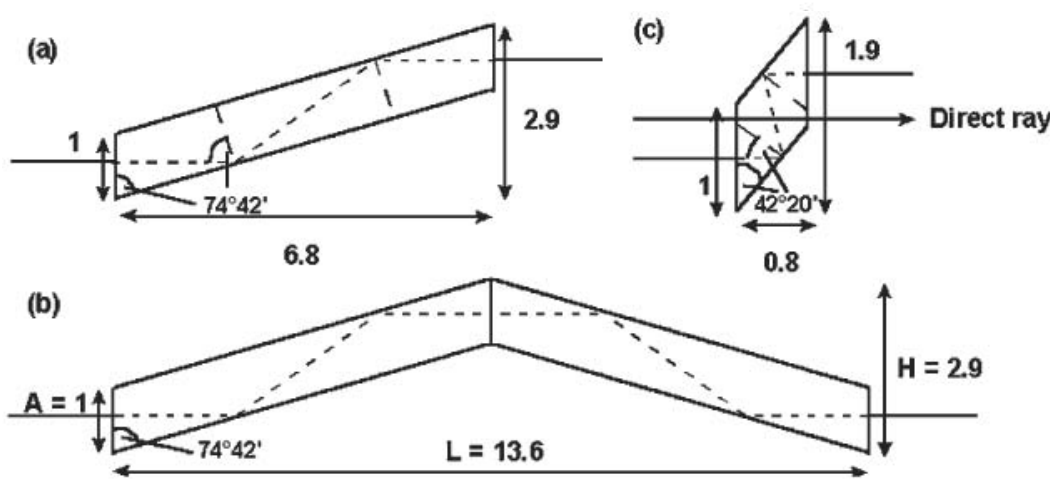

Figure 3. (a) Optical schematic of the (a) proposed $\lambda / 8$, (b) proposed $\lambda / 4$, and (c) unexplored $\lambda / 8$ rhombs. and a height of $2.3 \mathrm{~cm}$, as indicated by the dotted "standard" line at $\theta_{i}=54^{\circ} 37^{\prime}$ (Fig. 1b).

Figure $2 c$ describes the steeper $\lambda / 4$ rhomb with $\theta_{i}=48^{\circ} 37^{\prime}$, a typical aperture of $1 \mathrm{~cm}$, shorter length of $1.3 \mathrm{~cm}$, and height of $2.1 \mathrm{~cm}$, which would fit into a crowded system easier. But since the angular tolerance of $\theta_{\mathrm{i}}=48^{\circ} 37^{\prime}$ (with $\mathrm{d} \delta / \mathrm{d} \theta_{i}=53$ ) is smaller than $\theta_{i}=54^{\circ} 37^{\prime}$ (with $\mathrm{d} \delta / \mathrm{d} \theta_{i}=$ $-27)$, the $\theta_{i}=48^{\circ} 37^{\prime}$ configuration is not realized.

The standard $\lambda / 2$ rhomb is shown in Figure $2 b$ and is composed of two $\lambda / 4$ rhombs. Four reflections within a pair of $\lambda / 4$ rhombs produces a $\lambda / 2$ retarder. The output beam is colinear with the input beam, and the optical alignment is very flexible. For a standard $\lambda / 2$ rhomb, a typical aperture size of $1 \mathrm{~cm}$ requires a length of $3.7 \mathrm{~cm}$ and a height of $2.3 \mathrm{~cm}$, as indicated by the dotted "standard" line at $\theta_{i}=54^{\circ} 37^{\prime}$ (see Fig. 1b).

\section{The proposed $\lambda / 8$ and $\lambda / 4$ rhombs}

As indicated by the dashed lines labeled $\lambda / 8$ in Figure la, a phase shift of $\delta=22.5^{\circ}$ occurs at $\theta_{i}=42^{\circ} 20^{\prime}$ (with $\mathrm{d} \delta / \mathrm{d} \theta_{i}$ $=783$ ) and $\theta_{i}=74^{\circ} 42^{\prime}$ (with $\mathrm{d} \delta / \mathrm{d} \theta_{i}=-81$ ). Since the angular tolerance of $\theta_{i}=74^{\circ} 42^{\prime}$ is higher than $\theta_{i}=42^{\circ} 20^{\prime}$, the proposed $\lambda / 8 \mathrm{rhomb}$ uses two $\delta=22.5^{\circ} \mathrm{TIR}$ at $\theta_{i}=74^{\circ} 42^{\prime}$ (see Fig. 3a). The rhomb angle is equal to the incidence angle of $\theta_{i}=74^{\circ} 42^{\prime}$. The output beam is displaced laterally, parallel to the input beam. For the proposed $\lambda / 8 \mathrm{rhomb}$, a typical aperture size of $1 \mathrm{~cm}$ requires a length of $6.8 \mathrm{~cm}$ and a height of $2.9 \mathrm{~cm}$, as indicated by the dashed "proposed" line at $\theta_{i}=74^{\circ} 42^{\prime}$ (see Fig. 1b).

Figure $3 c$ describes the steeper $\lambda / 8$ rhomb with $\theta_{i}=42^{\circ} 20^{\prime}$, a typical aperture of $1 \mathrm{~cm}$, shorter length of $0.8 \mathrm{~cm}$, and a height of $1.9 \mathrm{~cm}$, which would fit into a crowded system easier. But since the angular tolerance of the $\theta_{i}=42^{\circ} 20^{\prime}$ (with $\mathrm{d} \delta / \mathrm{d} \theta_{i}=783$ ) is smaller than the angular tolerance of $\theta_{i}=74^{\circ} 42^{\prime}$ (with $\mathrm{d} \delta / \mathrm{d} \theta_{i}=-81$ ), the $\theta_{i}=$ $42^{\circ} 20^{\prime}$ configuration is not explored. Also since $42^{\circ} 20^{\prime}$ is smaller than $45^{\circ}$, the $\theta_{i}=42^{\circ} 20^{\prime}$ configuration allows the transmission of direct rays as indicated in Figure 3c.

The proposed $\lambda / 4$ rhomb is shown in Figure $3 \mathrm{~b}$ and is composed of two $\lambda / 8$ rhombs. The output beam is colinear with the input beam (similar to the case of the standard $\lambda / 2$ rhomb) making optical alignment very flexible. For the proposed $\lambda / 4$ rhomb, a typical aperture size of $1 \mathrm{~cm}$ requires a length of $13.6 \mathrm{~cm}$ and a height of $2.9 \mathrm{~cm}$, as indicated by the dashed "proposed" line at $\theta_{i}=74^{\circ} 42^{\prime}$ (see Fig. $1 b)$. For the same aperture size the proposed $\lambda / 4$ rhomb with colinear output beam has 7.4 times the length and $1.3 \mathrm{x}$ the height of the standard $\lambda / 4$ rhomb. The angular tolerance of the proposed $\lambda / 4$ rhomb (with $\mathrm{d} \delta / \mathrm{d} \theta_{i}=-81$ ) is only $3 \times$ lower than the standard $\lambda / 4$ rhomb (with $\mathrm{d} \delta / \mathrm{d} \theta_{i}=-27$ ). Therefore, for applications where colinearity is an important factor, the proposed $\lambda / 4$ rhomb is a good candidate for replacing the standard $\lambda / 4$ rhomb.

\section{References}

1. M. Born and E. Wolf, Principles of Optics (Pergamon Press, Oxford, U.K., 1987) p. 50. 